\title{
Photonic glass ceramics based on SnO2 nanocrystals: advances and perspectives
}

\section{Tran, Lam Thi Ngoc, Armellini, Cristina, Balda, Rolindes, Benabdesselam, Mourad, Berneschi, Simone, et al.}

Lam Thi Ngoc Tran, Cristina Armellini, Rolindes Balda, Mourad Benabdesselam, Simone Berneschi, Wilfried Blanc, Brigitte Boulard, Alessandro Carpentiero, Andrea Chiappini, Alessandro Chiasera, Paola Dentella, Dominik Dorosz, Shane Eaton, Mario Christian Falconi, Joaquin Fernandez, Maurizio Ferrari, James Gates, Pawel Gluchowski, Gloria Ischia, Anna Lukowiak, Franck Mady, Damiano Massella, Gualtiero Nunzi Conti, Francesco Prudenzano, Barbara Rossi, Roberta Ramponi, Giancarlo C. Righini, Pier-John Sazio, Giorgio Speranza, Stefano Varas, Daniele Zonta, Lidia Zur, "Photonic glass ceramics based on $\mathrm{SnO} 2$ nanocrystals: advances and perspectives," Proc. SPIE 11276, Optical Components and Materials XVII, 1127614 (3 March 2020); doi: 10.1117/12.2547526 


\section{Photonic glass ceramics based on $\mathrm{SnO}_{2}$ nanocrystals: advances and perspectives}

Lam Thi Ngoc Tran ${ }^{\mathrm{a}}$, Cristina Armellini ${ }^{\mathrm{b}}$, Rolindes Balda ${ }^{\mathrm{c}, \mathrm{d}}$, Mourad Benabdesselam ${ }^{\mathrm{e}}$, Simone Berneschi $^{\mathrm{f}}$, Wilfried Blanc ${ }^{\mathrm{e}}$, Brigitte Boulard ${ }^{\mathrm{g}}$, Alessandro Carpentiero $^{\mathrm{b}}$, Andrea Chiappini ${ }^{\mathrm{b}}$, Alessandro Chiasera ${ }^{\mathrm{b}}$, Paola Dentella ${ }^{\mathrm{h}}$, Dominik Dorosz ${ }^{\mathrm{i}}$, Shane Eaton ${ }^{\mathrm{j}}$, Mario Christian Falconi ${ }^{\mathrm{k}}$, Joaquin Fernandez ${ }^{1}$, Maurizio Ferrari ${ }^{*}, \mathrm{~b}, \mathrm{~m}$, James Gates $^{\mathrm{n}}$, Pawel Gluchowski ${ }^{\mathrm{o}}$, Gloria Ischia ${ }^{\mathrm{p}}$, Anna Lukowiak $^{\circ}$, Franck Madye, Damiano Massella ${ }^{\mathrm{q}}$, Gualtiero Nunzi Conti ${ }^{\mathrm{f}}$, Francesco Prudenzano ${ }^{\mathrm{k}}$, Barbara Rossi ${ }^{\mathrm{r}}$, Roberta Ramponi ${ }^{\mathrm{j}}$, Giancarlo C. Righini ${ }^{\mathrm{m}, \mathrm{f}}$, Pier-John Sazio ${ }^{\mathrm{n}}$, Giorgio Speranza ${ }^{\mathrm{s}}$, Stefano Varas ${ }^{\mathrm{b}}$, Daniele Zonta ${ }^{\mathrm{t}, \mathrm{u}, \mathrm{b}}$ and Lidia Zur ${ }^{\mathrm{b}}$

a Department of Materials Technology, Faculty of Applied Sciences, Ho Chi Minh City University of

Technology and Education, Vo Van Ngan street 1, Linh Chieu Ward, Thu Duc District, Ho Chi Minh City, Vietnam

${ }^{b}$ IFN-CNR CSMFO Lab. and FBK Photonics Unit, Via alla Cascata 56/C Povo 38100 Trento, Italy

'Departamento de Física Aplicada I, Escuela de Ingeniería de Bilbao, Universidad del País Vasco

UPV-EHU, Plaza Ingeniero Torres Quevedo 148013 Bilbao, Spain

${ }^{\mathrm{d}}$ Materials Physics Center CSIC-UPV/EHU, 20018 San Sebastian, Spain

${ }^{\text {e} U n i v e r s i t e ́ ~ C o ̂ t e ~ d ' A z u r, ~ I n s t i t u t ~ d e ~ P h y s i q u e ~ d e ~ N i c e, ~ C N R S ~ U M R 7010, ~ P a r c ~ V a l r o s e, ~} 06018$ Nice

Cedex 2, France

fIFAC - CNR, Sesto Fiorentino, Italy

'IMMM UMR CNRS 6283, Université. du Maine, Le Mans, France

hDepartment of Physics, Politecnico di Milano, p.zza Leonardo da Vinci 32, 20133 Milano, Italy

${ }^{i}$ AGH University of Science and Technology, 30 Mickiewicza Av., 30-059 Krakow, Poland

${ }^{j}$ IFN-CNR and Department of Physics, Politecnico di Milano, p.zza Leonardo da Vinci 32, 20133

Milano, Italy

kPolitecnico di Bari - Via Amendola 126/b - 70126 Bari, Italy

'Donostia International Physics Center DIPC, 20018 San Sebastian, Spain

museo Storico della Fisica e Centro Studi e Ricerche "Enrico Fermi", Piazza del Viminale 1, 00184 Roma, Italy

${ }^{\mathrm{n} O R C}$, Optoelectronics Research Centre, University of Southampton, University Road, Southampton SO17 1BJ, UK

${ }^{\circ}$ Institute of Low Temperature and Structure Research, PAS, Wroclaw, Poland

PDepartment of Industrial Engineering, University of Trento, Via Sommarive 9, 38123 Trento, Italy

qUniversity of Vigo, EI Telecomunicacion - Campus Universitario As Lagoas 36310 Vigo, Spain

${ }^{\mathrm{r}}$ Elettra - Sincrotrone Trieste, Trieste, Italy

${ }^{\mathrm{s}}$ FBK CMM- FMPS Unit, Via Sommarive 18, Povo, Trento, 38123, Italy

tDepartment of Civil, Environmental and Mechanical Engineering, University of Trento, Mesiano-

Trento, Italy

uDepartment of Civil and Environmental Engineering, University of Strathclyde, Glasgow, G11XJ, UK

* maurizio.ferrari@ifn.cnr.it; Tel: +39 0461 314918; fax +39 0461 314875; http://www.tn.ifn.cnr.it/ 


\begin{abstract}
$\mathrm{SnO}_{2}$-based glass-ceramics activated by rare earth ions have been extensively investigated because of the need to develop reliable fabrication protocols and clarify some interesting optical, structural, and spectroscopic features of the system. There is one important weakness in glass photonics when the rare earth ions are employed as luminescent sources. This is the low absorption cross section of the electronic states of the rare earth ions. A sensitizer is therefore requested. In the last years, we demonstrated that $\mathrm{SiO}_{2}-\mathrm{SnO}_{2}$ glass ceramics, presenting a strong absorption cross section in the UV range due to the $\mathrm{SnO}_{2}$ nanocrystal, are effective rare earth ions sensitizers. Another interesting property of the $\mathrm{SiO}_{2}-\mathrm{SnO}_{2}$ system is its photorefractivity. The high photorefractivity of sol-gel-derived $\mathrm{SnO}_{2}-\mathrm{SiO}_{2}$ glass-ceramic waveguides has been demonstrated in several papers published by our consortium. It has been shown that the UV irradiation induces refractive index change allowing the direct writing of both channel waveguides and Bragg gratings.

The results presented in this communication not only demonstrate the viability and outstanding properties of the $\mathrm{SiO}_{2}$ $\mathrm{SnO}_{2}$ glass-ceramics for photonic applications but also put the basis for the fabrication of solid state and integrated lasers. The next steps of the research are the fabrication of the channels and mirrors exploiting the photorefractivity as well as to draw glass ceramic fiber, checking the lasing action and corresponding functional characteristics. Finally, it is worth noting that the dynamic of the energy transfer from the nanocrystals to the rare earth ions is still an exciting open question.
\end{abstract}

Keywords: $\mathrm{SiO}_{2}-\mathrm{SnO}_{2}$, photonic glass-ceramics, rare earth ions, luminescence sensitizers, photorefractivity, sol-gel technology, glass photonics, nanocrystals.

\title{
1. INTRODUCTION
}

In the current Age of Light, Photonics is a key enabling technology ${ }^{1}$ for different segments: life science and health, industrial manufacturing and quality, information and communication, emerging light, electronics and displays. And, for the development of optical devices, i.e. the heart elements of photonic applications, glass-based rare-earth-activated optical structures play an essential role ${ }^{2}$. In this area of glass photonics, photonic glass-ceramics have become a strategic choice because they combine the optical and glass-manufacturing processing properties of the amorphous phase and the single-crystal-like optical and spectroscopic properties of the crystalline phases, which is important in luminescent materials activated species, e.g. rare-earths ${ }^{3-6}$. Following this strategy, photonic glass-ceramics based on $\mathrm{SnO}_{2}$ nanocrystals have drawn much special attention since they exhibit two innovative and unique characteristics: (i) luminescence sensitizing and (ii) photorefractivity. Furthermore, $\mathrm{SnO}_{2}$ based glass-ceramics gives a solution to two crucial points which are decisive in the development of an optically pumped rare-earth-based laser: (i) the low absorption cross section of the rare-earth ions; (ii) the writing of channels and mirrors in the case of waveguide integrated laser. The role of $\mathrm{SnO}_{2}$ nanocrystals as rare-earth ion luminescence sensitizers allows to overcome the low absorption cross section of the $\mathrm{Er}^{3+}$ ion ${ }^{7,8}$. The photorefractivity of sol-gel derived $\mathrm{SnO}_{2}$ glass-ceramics allows applying the robust direct laser photoinscription technique on the systems to fabricate Bragg gratings and channel waveguides for waveguide integrated laser $^{9,10,11}$. The results presented in this communication not only demonstrate the viability and outstanding properties of the $\mathrm{SiO}_{2}-\mathrm{SnO}_{2}$ glass-ceramics for photonic applications but also put the basis for the fabrication of solid state and integrated lasers.

Considering the limitation of low $\mathrm{SnO}_{2}$ content or the presence of nonstoichiometric $\mathrm{SnO}_{\mathrm{x}}$ encountered by several fabrication methods (MCVD, melt-quenching and ion-implantation), sol-gel has been a profitable alternative to produce $\mathrm{SiO}_{2}-\mathrm{SnO}_{2}$ photonic glass-ceramics with higher $\mathrm{SnO}_{2}$ content thanks to its low temperature and melt-free synthesis ${ }^{2,5}$. High content of $\mathrm{SnO}_{2}$ nanocrystals in the sol-gel derived $\mathrm{SiO}_{2}-\mathrm{SnO}_{2}$ glass-ceramic systems gives advantages not only to obtain effective rare earth luminescence sensitization but also high photorefractivity as indicated in our recent works $^{8,11,12}$. However, to achieve this, the first research demand is to develop reliable fabrication protocols and controlling the ion-ion interaction ${ }^{7,13}$. In this work, we show the viability of sol-gel technology for fabricating transparent 
$\mathrm{SiO}_{2}-\mathrm{SnO}_{2}$ glass-ceramics in different forms of monoliths and planar waveguides containing high $\mathrm{SnO}_{2}$ nanocrystal contents in silica matrix: $10 \mathrm{~mol} \%$ for the monoliths and $30 \mathrm{~mol} \%$ for the planar waveguides. Afterwards, by the spectroscopic properties of the $90 \mathrm{SiO}_{2}-10 \mathrm{SnO}_{2}: 0.5 \mathrm{Er}^{3+}$ monoliths, we confirm the effective role of $\mathrm{SnO}_{2}$ nanocrystal as $\mathrm{Er}^{3+}$ luminescence sensitizer. Furthermore, our recent results on the photorefractivity of the sol-gel derived $\mathrm{SiO}_{2}-\mathrm{SnO}_{2}$ glass-ceramics are presented in comparison with other substitutional tin-doped silica glasses showing the viability of the development of not only Bragg gratings and channel waveguides but also active optical integrated devices, e.g. light sources and monolithic optical integrated circuits based on REs-doped $\mathrm{SiO}_{2}-\mathrm{SnO}_{2}$ glass-ceramics.

\section{FABRICATION PROCESSES}

\subsection{Sol-gel derived fabrication processes for $\mathrm{SiO}_{2}-\mathrm{SnO}_{2}$ glass-ceramics}

In this session, the fabrication of $\mathrm{SiO}_{2}-\mathrm{SnO}_{2}: \mathrm{Er}^{3+}$ planar waveguides and monoliths using sol-gel method is described. In general, both sol-gel routes used for the planar waveguides and monoliths fabrication started from the solution syntheses in which all hydrolysis and condensation reactions could occur. Concerning the chemical reactants, tetraethyl orthosilicate (TEOS), tin(II) chloride $\left(\mathrm{SnCl}_{2} .2 \mathrm{H}_{2} \mathrm{O}\right)$ and erbium(III) nitrate pentahydrate $\left(\mathrm{Er}\left(\mathrm{NO}_{3}\right)_{3} .5 \mathrm{H}_{2} \mathrm{O}\right)$ were used as the precursors of $\mathrm{SiO}_{2}, \mathrm{SnO}_{2}$ and $\mathrm{Er}^{3+}$ respectively and $\mathrm{HCl}$ was used as a catalyst to promote the reactions.

At the beginning, all these precursors were dissolved in Ethanol (EtOH) and mixed together. Then, a proper amount of de-ionized water containing $\mathrm{HCl}$ catalyst was poured into the mixture to proceed the hydrolysis and condensation reaction to obtain the final solution. The quantities of all the chemicals used for the $\mathrm{SiO}_{2}-\mathrm{SnO}_{2}: \mathrm{Er}^{3+}$ syntheses were calculated based on the fundamental ratios of $\mathrm{TEOS} / \mathrm{H}_{2} \mathrm{O} / \mathrm{HCl}$ which were $1 / 10 / 0.009$ for the monoliths ${ }^{14,15}$ and $1 / 2 / 0.0037$ for the planar waveguides ${ }^{8,11,12}$.

Afterwards, depending on the structural geometry of each system, the viscosity, reactant ratios and treatment for the solutions were established specifically. In monoliths, to obtain 3D structure xerogel, the synthesized solutions were transferred into molds and left to finish aging, being dried and eventually heat-treated. In planar waveguides, i.e. 2Dstructures, for the dip-coating deposition, low viscosity of the solutions for the waveguides was required ${ }^{11,16}$ which was much lower than the ones for the monoliths and thus, the aging time was also limited in several hours. The solutions then are used for dip-coating to deposit the planar waveguides. The chemicals and synthesized solutions played principal role in defining texture and consequent properties of the final sol-gel derived products. Therefore, the development of synthesis protocols, with appropriate thermal treatment was the first demand to obtain homogeneous and transparent $\mathrm{SiO}_{2}-\mathrm{SnO}_{2}$ glass-ceramics.

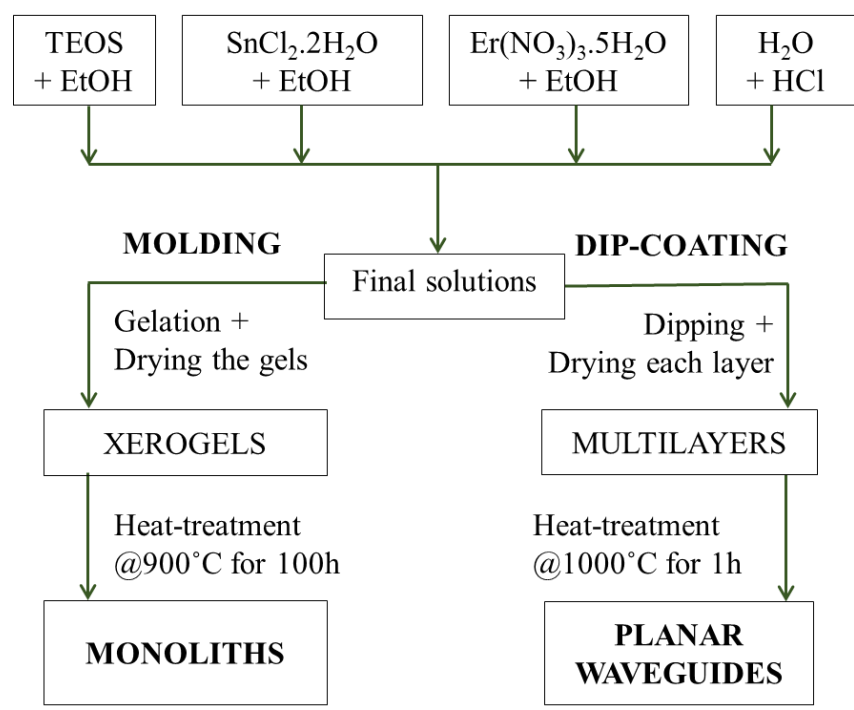

Figure 1. Flow chart of sol-gel derived fabrication processes of $\mathrm{SiO}_{2}-\mathrm{SnO}_{2}: \mathrm{Er}^{3+}$ glass-ceramic monoliths and planar waveguides 
Concerning the heat-treatment, after the continuously consolidated works ${ }^{14,15,11,12,8}$, optimal heat-treatment processes for obtaining optically qualified $\mathrm{SiO}_{2}-\mathrm{SnO}_{2}: \mathrm{Er}^{3+}$ glass-ceramic systems were defined. Based on the study on thermal analyses ${ }^{14,15}$, a multi-step heat-treatment up to $900^{\circ} \mathrm{C}$ for a prolonged annealing time, e.g. $100 \mathrm{~h}^{12,11,8}$ has recently shown the best performance in terms of water removal and densification for the monoliths. Referring to the planar waveguides, the optimal heat-treatment was $1000^{\circ} \mathrm{C}$ for $1 \mathrm{~h}^{11,12}$. Briefly, the fabrication protocols for both $\mathrm{SiO}_{2}-\mathrm{SnO}_{2}: \mathrm{Er}^{3+}$ glass ceramic monoliths and planar waveguides are described in Figure 1.

\subsection{Obtained $\mathrm{SiO}_{2}-\mathrm{SnO}_{2}$ glass-ceramics}

Sol-gel technology provides versatility of fabricating multicomponent materials with controlled composition, shape, morphological and textual properties of the final products ${ }^{8,11,12}$. One can see that from Table 1 which summarizes all the details concerning the composition $\left(\mathrm{SnO}_{2}\right.$ content and $\mathrm{Er}^{3+}$ dopant concentration), and geometry of the $\mathrm{SiO}_{2}-\mathrm{SnO}_{2}: \mathrm{Er}^{3+}$ glass-ceramic monoliths and planar waveguides obtained by sol-gel fabrication protocols described in Figure 1 .

Table 1. Composition and geometrical details of the fabricated transparent $\mathrm{SiO}_{2}-\mathrm{SnO}_{2}: \mathrm{Er}^{3+}$ glass-ceramic systems including monoliths and planar waveguides

\begin{tabular}{|c|c|c|c|}
\hline \multirow[b]{2}{*}{ System } & \multicolumn{2}{|c|}{ Composition $(100-\mathrm{x}) \mathrm{SiO}_{2}-\mathrm{xSnO}_{2}: \mathrm{yEr}^{3+}$} & \multirow[b]{2}{*}{$\begin{array}{l}\text { Geometrical details of fabricated transparent } \mathrm{SiO}_{2-} \\
\qquad \mathrm{SnO}_{2} \text { glass-ceramics }\end{array}$} \\
\hline & $\begin{array}{l}\mathrm{SnO}_{2} \text { content } \\
\mathrm{x}(\mathrm{mol} \%)\end{array}$ & $\begin{array}{l}\mathrm{Er}^{3+} \text { concentration } \\
\mathrm{n}_{\mathrm{SiO}_{2}}+\mathrm{n}_{\mathrm{SnO}_{2}}\end{array}$ & \\
\hline Monoliths & $0,5,7.5$ and 10 & $0.25,0.50,0.75$ and 1.00 & $\begin{array}{l}\text { - Monolithic squares: } 1 \times 1 \mathrm{~cm}^{2} \text { cross section and } \\
0.3 \mathrm{~cm} \text { height } \\
\text { - Cylinders: } 0.4 \mathrm{~cm} \text { diameter and } 1.5 \mathrm{~cm} \text { height } \\
\text { - Big pillars: } 1 \mathrm{~cm} \text { diameter and } 3.3 \mathrm{~cm} \text { height }\end{array}$ \\
\hline $\begin{array}{c}\text { Planar } \\
\text { waveguides }\end{array}$ & $\begin{array}{l}0,5,10,15,20 \\
25 \text { and } 30\end{array}$ & $0.5,1.0$ and 1.5 & Thickness: $\sim 1.2 \mu \mathrm{m}$ \\
\hline
\end{tabular}

From Table 1, we have demonstrated that sol-gel is a profitable route for obtaining transparent $\mathrm{SiO}_{2}-\mathrm{SnO}_{2}$ glass-ceramics containing high $\mathrm{SnO}_{2}$ nanocrystal contents: up to $10 \mathrm{~mol} \%$ for the monoliths and $30 \mathrm{~mol} \%$ for the planar waveguides. After the final heat-treatment, i.e. $900^{\circ} \mathrm{C}$ for $100 \mathrm{~h}$ in case of the monoliths and $1000^{\circ} \mathrm{C}$ for $1 \mathrm{~h}$ in case of the planar waveguides, all the glass-ceramics are crack-free and densified. Furthermore, these sol-gel protocols allow controlling the size of $\mathrm{SnO}_{2}$ nanocrystals of less than $10 \mathrm{~nm}$ as demonstrated in the structural characterization in our recent publication $^{12}$. All obtained compositional planar waveguides have thickness of around $1.2 \mu \mathrm{m}$ and with this thickness, the $\mathrm{SiO}_{2}-\mathrm{SnO}_{2}$ glass-ceramic planar waveguides containing higher than $10 \mathrm{~mol} \% \mathrm{SnO}_{2}$ nanocrystals supports single TE and TM mode propagation at $1.5 \mu \mathrm{m}$. Different geometries of the monoliths were fabricated with the attention to the oriented applications. The monolithic squares (cross section of $1 \times 1 \mathrm{~cm}^{2}$ and height of $0.3 \mathrm{~cm}$ ) were produced for writing channel waveguides.

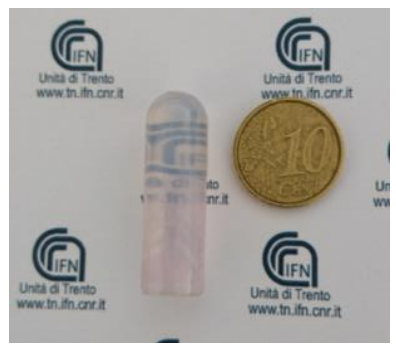

Figure 2. Photo of the fabricated $95 \mathrm{SiO}_{2}-5 \mathrm{SnO}_{2}: 0.5 \mathrm{Er}^{3+}$ big pillar $(1 \mathrm{~cm}$ diameter and $3.3 \mathrm{~cm}$ length $)$ obtained after heat-treatment at $900{ }^{\circ} \mathrm{C}$ for $100 \mathrm{~h}^{11}$. 


\section{3. $\mathrm{SnO}_{2}$ NANOCRYSTAL AS AN EFFECTIVE Er $^{3+}$ LUMINESCENCE SENSITIZER}

With the strong absorption cross section in the $\mathrm{UV}, \mathrm{SnO}_{2}$ nanocrystals has been demonstrated to be effective sensitizers for the rare-earth luminescence ${ }^{7,8,11}$. The strategy of exploiting $\mathrm{SnO}_{2}$ nanocrystals as rare earth luminescence sensitizers provides the solution for the low absorption cross section issue of the electronic states of the rare earth ions arising from the parity-forbidden $4 \mathrm{f}-4 \mathrm{f}$ transitions with naturally weak intensity ${ }^{2,8}$. In Figure $3 \mathrm{a}$, the absorption spectrum in the UVVis region of the $90 \mathrm{SiO}_{2}-10 \mathrm{SnO}_{2}: 0.5 \mathrm{Er}^{3+}$ monoliths showed an optical absorption band edge centered at around $3.5 \mathrm{eV}$ $(\sim 340 \mathrm{~nm})$ corresponding to the UV absorption of the interband transition of $\mathrm{SnO}_{2}$ nanocrystals ${ }^{7,17,18}$. Several absorption bands corresponding to the transitions of $\mathrm{Er}^{3+}$ from the ground state ${ }^{4} \mathrm{I}_{15 / 2}$ to other excited states such as ${ }^{4} \mathrm{G}_{11 / 2},{ }^{4} \mathrm{~F}_{5 / 2},{ }^{4} \mathrm{~F}_{7 / 2}$, ${ }^{2} \mathrm{H}_{11 / 2},{ }^{4} \mathrm{~S}_{2 / 3}$ and ${ }^{4} \mathrm{~F}_{9 / 2}$ are also assigned. From this figure, one can see that the UV interband absorption of $\mathrm{SnO}_{2}$ nanocrystals is much stronger than the absorption bands corresponding to the transitions of $\mathrm{Er}^{3+}$ from the ground state ${ }^{4} \mathrm{I}_{15 / 2}$ to the other excited states. Therefore, from Figure $3 \mathrm{~b}$, i.e. the excitation spectrum of the $90 \mathrm{SiO}_{2}-10 \mathrm{SnO}_{2}: 0.5 \mathrm{Er}^{3+}$ monoliths obtained by recording the luminescence signal at $1530 \mathrm{~nm}$, the fingerprint of the ${ }^{4} \mathbf{I}_{13 / 2} \rightarrow{ }^{4} \mathrm{I}_{15 / 2}$ transition of $\mathrm{Er}^{3+}$, the more intense $1530 \mathrm{~nm}$ emission from the $\mathrm{Er}^{3+}$ metastable state ${ }^{4} \mathrm{I}_{13 / 2}$ is evidently achieved by exciting in the $\mathrm{SnO}_{2}$ band gap at around $340 \mathrm{~nm}$ in comparison with directly exciting $\mathrm{Er}^{3+}$ to its electronic states such as ${ }^{4} \mathrm{~F}_{5 / 2},{ }^{4} \mathrm{~F}_{7 / 2}$, ${ }^{2} \mathrm{H}_{11 / 2},{ }^{2} \mathrm{~F}_{9 / 2}$ and ${ }^{4} \mathrm{I}_{11 / 2}$. These results demonstrate the role of $\mathrm{SnO}_{2}$ as an effective $\mathrm{Er}^{3+}$ luminescence sensitizer as well as the promising of an efficient laser exploiting this luminescence sensitization as a pumping scheme.

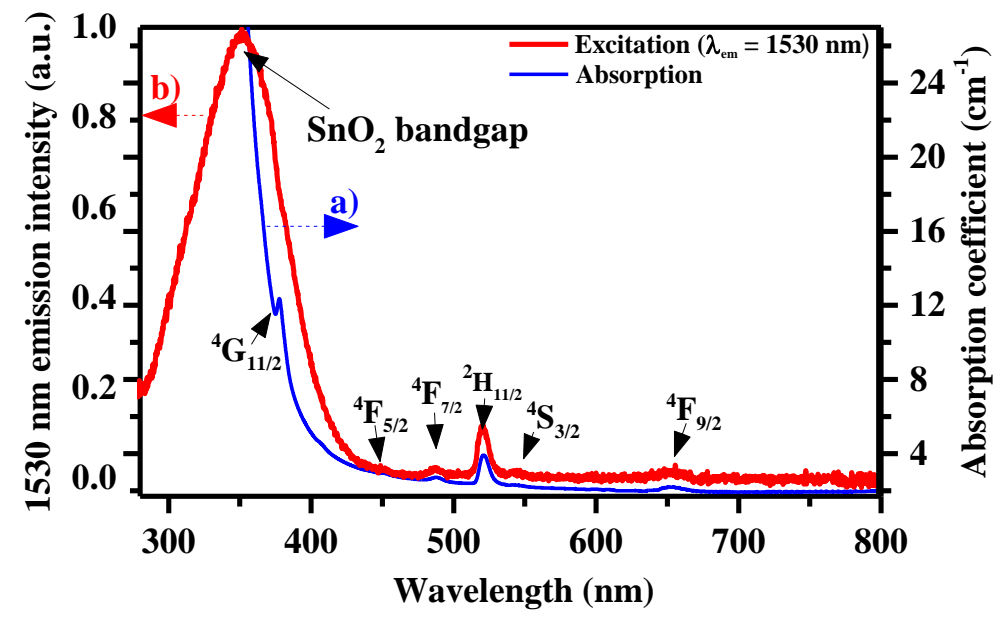

Figure 3. a) Room temperature absorption spectrum (blue line) and b) room temperature excitation spectrum (red line) obtained by detecting at $1530 \mathrm{~nm}$ emission of the $90 \mathrm{SiO}_{2}-10 \mathrm{SnO}_{2}: 0.5 \mathrm{Er}^{3+}$ glass-ceramic monolith. The $\mathrm{SnO}_{2}$ nanocrystal bandgap absorption and the electronic transitions from the ground state ${ }^{4} \mathrm{I}_{15 / 2}$ of the $\mathrm{Er}^{3+}$ ion are indicated.

The $1.5 \mu \mathrm{m}$ emission spectrum of the $90 \mathrm{SiO}_{2}-10 \mathrm{SnO}_{2}: 0.5 \mathrm{Er}^{3+}$ glass-ceramic monolith under the excitation at $340 \mathrm{~nm}$ in the $\mathrm{SnO}_{2}$ bandgap shown in Figure 4 which exhibits Stark splitting and narrow peaks confirms (i) the energy transfer from $\mathrm{SnO}_{2}$ nanocrystals to $\mathrm{Er}^{3+}$ ions and (ii) the evidence of $\mathrm{Er}^{3+}$ ions locating in the crystalline environment, i.e. $\mathrm{SnO}_{2}$ nanocrystals $711,12,15$. Although the mechanism of energy transfer from $\mathrm{SnO}_{2}$ to $\mathrm{Er}^{3+}$ can be proposed to be mediated by exciton and defects as in the works ${ }^{13,19,8}$, the dynamic of the energy transfer from the nanocrystal to the rare earth ion is still an exciting open question which needs to be clarified by detailed sets of experiments. 


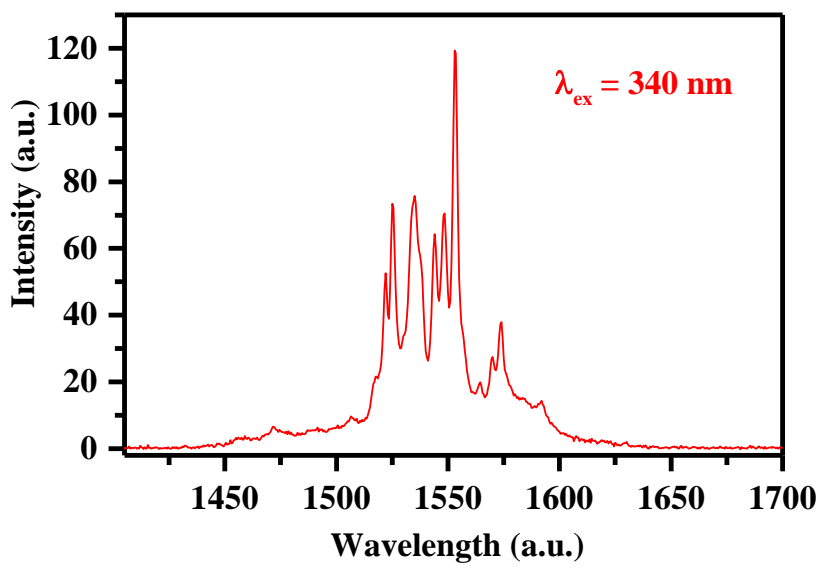

Figure 4. Room temperature ${ }^{4} \mathrm{I}_{13 / 2} \rightarrow{ }^{4} \mathrm{I}_{15 / 2}$ emission spectrum of $\mathrm{Er}^{3+}$ ions of the $90 \mathrm{SiO}_{2}-10 \mathrm{SnO}_{2}: 0.5 \mathrm{Er}^{3+}$ glass-ceramic monolith obtained upon excitation at $340 \mathrm{~nm}$ in the $\mathrm{SnO}_{2}$ nanocrystal bandgap.

\section{HIGH PHOTOREFRACTIVITY OF $\mathrm{SiO}_{2}-\mathrm{SnO}_{2}$ GLASS-CERAMICS $^{-}$}

The photorefractivity potential of tin dioxide-based transparent materials was first realized in tin-codoped germanosilicate $^{20,21}$ and phosphosilicate ${ }^{21,22}$ optical fibres. By introducing tin in such silicate glasses, strong photorefractive gratings with enhanced refractive index change up to $\sim 1.2-1.4 \times 10^{-3}$ were obtained. Thanks to Sn presence, the refractive index change was even 3 times larger than in the case of pure germanosilicate ${ }^{20,21}$. Therefore, the photorefractivity of the binary tin dioxide-silica materials has attracted numerous of researches ${ }^{8,9,11}$.

In substitutional tin-doped silica glasses, in which a very small content of tin (less than 0.5 mol $\%^{23}$ ) was introduced into silica, the refractive index change is positive and in the order of $10^{-4}$. The photorefractivity was demonstrated to relate to laser induced bleaching of the Sn-oxygen deficient centres (ODC) absorption ${ }^{24}$, and $\mathrm{Sn}_{-}\left(\mathrm{SiO}_{4}\right)_{\mathrm{n}}$ rings structural units of which the dimensions were reduced during UV exposure ${ }^{25-28}$.

Table 2. Photorefractivity of $\mathrm{SiO}_{2}-\mathrm{SnO}_{2}$ binary materials

\begin{tabular}{|c|c|c|c|c|c|}
\hline \multirow{2}{*}{$\begin{array}{c}\mathrm{SnO}_{2} \text { content } \\
(\mathrm{mol} \%)\end{array}$} & \multirow{2}{*}{$\begin{array}{l}\text { Fabrication } \\
\text { method }\end{array}$} & \multicolumn{2}{|c|}{ Irradiation process } & \multirow{2}{*}{$\begin{array}{c}\text { Induced refractive } \\
\text { index change } \\
(\Delta \mathrm{n})\end{array}$} & \multirow[b]{2}{*}{ The work } \\
\hline & & UV laser & $\begin{array}{l}\text { Cumulative } \\
\text { fluence }\end{array}$ & & \\
\hline 0.15 & MCVD & $248 \mathrm{~nm} \mathrm{KrF}$ excimer lasers & $20 \mathrm{~kJ} / \mathrm{cm}^{2}$ & $\Delta \mathrm{n}=+3.0 \times 10^{-4}$ & Brambilla et.al. $^{24}$ \\
\hline 0.4 & Sol-gel & $\begin{array}{c}266 \mathrm{~nm} \text { pulsed the } 4 \\
\text { harmonic Nd-YAG laser }\end{array}$ & $0.17 \mathrm{~kJ} / \mathrm{cm}^{2}$ & $\Delta \mathrm{n}=+4.0 \times 10^{-4}$ & Chiodini et.al. ${ }^{26}$ \\
\hline 5 & Sol-gel & $\begin{array}{c}266 \mathrm{~nm} \text { pulsed the } 4 \\
\text { harmonic Nd-YAG laser }\end{array}$ & $0.15 \mathrm{~kJ} / \mathrm{cm}^{2}$ & $\Delta \mathrm{n}=-6.0 \times 10^{-4}$ & Chiodini et.al. ${ }^{23,26}$ \\
\hline 25 & Sol-gel & $248 \mathrm{~nm} \mathrm{KrF}$ excimer lasers & $7.6 \mathrm{~kJ} / \mathrm{cm}^{2}$ & $\Delta \mathrm{n}=-1.6 \times 10^{-3}$ & $\begin{array}{l}\text { Berneschi et.al. }{ }^{10} \\
\text { Lukowiak et.al. }^{9}\end{array}$ \\
\hline 30 & Sol-gel & $248 \mathrm{~nm} \mathrm{KrF}$ excimer lasers & $0.3 \mathrm{~kJ} / \mathrm{cm}^{2}$ & $\Delta \mathrm{n}=-2.8 \times 10^{-3}$ & $\operatorname{Tran}^{11}$ \\
\hline
\end{tabular}


If the content of $\mathrm{SnO}_{2}$ in $\mathrm{SiO}_{2}$ matrix is high (more than $0.5 \mathrm{~mol} \%$ ), after proper heat-treatments, a segregation of $\mathrm{SnO}_{2}$ nanocrystals occurred and formed the tin dioxide-silicate glass-ceramics ${ }^{11,23}$. In this case, the photorefractivity of $\mathrm{SiO}_{2}-$ $\mathrm{SnO}_{2}$ glass-ceramics is demonstrated to be negative ${ }^{8-11,23}$. In the works ${ }^{8-10}$, the mechanism was suggested to involve two factors: (i) the volume expansion which is compensated by (ii) the material polarizability. However, due to the complexity and diversity of photorefractive manifestation depending on glass composition, fabrication history and irradiation condition, defining the origin of the photorefractivity of such glass-ceramics needs further experimental investigation to be completely understood. Nevertheless, we have demonstrated that the values of refractive index change of the sol-gel derived tin dioxide-based glass-ceramics are one order of magnitude higher than the ones of tindoped silica glasses, and they increase with the increasing $\mathrm{SnO}_{2}$ content in $\mathrm{SiO}_{2}$ matrix ${ }^{8,11}$ as evidenced from Table 2. In other words, the high $\mathrm{SnO}_{2}$-containing glass-ceramics that have been achieved using sol-gel technology give the advantage of greater UV induced refractive index changes. Moreover, with the high refractive index change in the order of $10^{-3}$, the sol-gel derived $\mathrm{SiO}_{2}-\mathrm{SnO}_{2}$ glass-ceramics are potential for the UV direct writing of channel waveguides and gratings. Therefore, it is compelling for the development of active optical integrated devices, e.g. light sources and monolithic optical integrated circuits based on REs-doped $\mathrm{SiO}_{2}-\mathrm{SnO}_{2}$ glass-ceramics ${ }^{8-11}$.

\section{CONCLUSION}

In summary, this work shows the consolidated results and recent advances in $\mathrm{Er}^{3+}$-activated $\mathrm{SnO}_{2}-\mathrm{SiO}_{2}$ transparent glassceramics, putting basis for the fabrication of solid state and integrated lasers. Concerning the fabrication, reliable sol-gel synthesis protocols with defined thermal processes have been developed to prepare both the compositional photonic monoliths and planar waveguides. The homogeneous and transparent glass-ceramic monoliths were obtained with $\mathrm{SnO}_{2}$ content up to $10 \mathrm{~mol} \%$. Three different bulk forms were produced for the desired applications: the cylinders for solid state laser construction, the monolithic squares for writing channel waveguides and big pillars for the usages for fiber preform preparation. Referring to the planar waveguides, high $\mathrm{SnO}_{2}$ content up to $30 \mathrm{~mol} \%$ was successfully introduced in $\mathrm{SiO}_{2}$ matrix. The spectroscopic measurements demonstrate: (i) energy transfer from $\mathrm{SnO}_{2}$ to $\mathrm{Er}^{3+}$ and (ii) the role of $\mathrm{SnO}_{2}$ nanocrystal as an effective $\mathrm{Er}^{3+}$ luminescence sensitizer as well as the promising of an efficient laser exploiting this luminescence sensitization as a pumping scheme. The photorefractivity investigation shows that the sol-gel derived $\mathrm{SiO}_{2}-\mathrm{SnO}_{2}$ glass-ceramics with high negatively induced refractive index change (in the order of $10^{-3}$ ) is viable for the UV direct writing of Bragg gratings and channel waveguides. With the two demonstrated unique characteristics, REs-doped $\mathrm{SiO}_{2}-\mathrm{SnO}_{2}$ glass-ceramics are compelling candidates for the development of active optical integrated devices, e.g. light sources and monolithic optical integrated circuits.

The consolidated steps of the research are fabricating the channels and mirrors exploiting the obtained photorefractivity with a proper pumping scheme and checking the lasing action and corresponding functional characteristics. Referring to the $\mathrm{SiO}_{2}-\mathrm{SnO}_{2}: 0.5 \mathrm{Er}^{3+}$ monoliths, the current results are reasonably qualified for the checking of lasing action with the designed lateral pumping scheme as a proof of concept. An elaboration for the big pillars preparation for fiber preforms and consequently, drawing the $\mathrm{SiO}_{2}-\mathrm{SnO}_{2}: \mathrm{Er}^{3+}$ glass-ceramic fibers is demanded. The dynamic of the energy transfer from the nanocrystals to the rare earth ions deserves further experiments to be completely explored.

\section{Acknowledgements}

This research is performed in the framework of the projects ERANet-LAC "RECOLA" (2017-2019), Centro Fermi MiFo (2017-2020) and NaWaGui (ANR-18-MRS1-0014). WB and MF acknowledge the support of CNR-STM - Short Term Mobility program 2019-2020.

\section{REFERENCES}

[1] Photonics21 Secretariat, "Europe's age of light! How photonics will power growth and innovation", Brussels, Düsseldorf, March 2019, <https://www.photonics21.org/download/ppp-services/photonics-downloads/Europes-age-oflight-Photonics-Roadmap-C1.pdf> (March 2019).

[2] Zur, L., Tran, L. T. N., Meneghetti, M., Varas, S., Armellini, C., Ristic, D., Chiasera, A., Scotognella, F., Pelli, S., Conti, G. N., Boulard, B., Zonta, D., Dorosz, D., Lukowiak, A., Righini, G. C., Ramponi, R., and Ferrari, M., "Glass and glass-ceramic photonic systems", Proc. SPIE 10106, Integrated Optics: Devices, Materials, and Technologies XXI, 1010603 (2017). 
[3] Boulard, B., Van, T. T. T., Łukowiak, A., Bouajaj, A., Gonçalves, R. R., Chiappini, A., Chiasera, A., Blanc, W., Duran, A., Turrell, S., Prudenzano, F., Scotognella, F., Ramponi, R., Marciniak, M., Righini, G. C., and Ferrari, M., "Photonic glass-ceramics: consolidated outcomes and prospects", Proc. of SPIE 9364, 93640Z-1/10 (2015).

[4] Pablos-Martin, A. de, Ferrari, M., Pascual, M.J., and Righini, G.C., "Glass-ceramics: A class of nanostructured materials for photonics", Rivista del Nuovo Cimento 38, 311-369 (2015).

[5] Gorni, G., Velázque, J. J., Mosa, J., Balda, R., Fernández, J., Durán, A., and Castro, Y., "Transparent Glass-Ceramics Produced by Sol-Gel: A Suitable Alternative for Photonic Materials", Materials 11, 212-1/30 (2018).

[6] Lukowiak, A., Vasilchenko, I., Normani, S., Chiappini, A., Chiasera, A., Armellini, C., Arfuso Duverger, C., Boulard, B., Wiglusz, R. J., Pelli, S., Battisha, I. K., Righini, G. C., Marciniak, M., and Ferrari, M., "Glass-ceramics for photonics: advances and perspectives" Proc. IEEE $16^{\text {th }}$ International Conference on Transparent Optical Networks ICTON, 1-4 (2014).

[7] Zur, L., Tran, T. N. L., Meneghetti, M., Tran, T. T. V., Lukowiak, A., Chiasera, A., Zonta, D., Ferrari, M., and Righini, G. C., "Tin-dioxide nanocrystals as $\mathrm{Er}^{3+}$ luminescence sensitizers: formation of glass-ceramics thin films and their characterization", Optical Materials 63, 95-100 (2017).

[8] Tran, L. T. N., Massella, D., Balda, R., Berneschi, S., Blanc, W., Boulard, B., Chiappini, A., Chiasera, A., Dentella, P., Eaton, S., Fernandez, J., Ferrari, M., Gates, J., Gluchowski, P., Ischia, G., Lukowiak, A., Conti, G. N., Prudenzano, F., Rossi, B., Righini, G. C., Zonta, D., and Zur, L., " $\mathrm{SiO}_{2}-\mathrm{SnO}_{2}$ photonic glass-ceramics", Proc. IEEE $21^{\text {st }}$ International Conference on Transparent Optical Networks ICTON, We.C6.1, 1-6 (2019).

[9] Lukowiak, A., Zur, L., Tran, T. N. L., Meneghetti, M., Berneschi, S., Conti G. N., Pelli, S., Trono, C., Bhaktha, B. N. S., "Sol-Gel-Derived Glass-Ceramic Photorefractive Films for Photonic Structures", Crystals, 7(2), 61 (2017).

[10] Berneschi S., Bhaktha B. N. S., Chiappini, A., Chiasera, A., Ferrari, M., Kinowski, C., Turrell, S., Trono, C., Brenci, M., Cacciari, I., Conti, G. N., Pelli, S., Righini, G. C., "Highly photorefractive $\mathrm{Eu}^{3+}$ activated sol-gel $\mathrm{SiO}_{2}-\mathrm{SnO}_{2}$ thin film waveguides", Proc SPIE 7604, 76040Z (2010).

[11] Tran, LTN, "Tin dioxide-based photonic glass-ceramics", Doctoral dissertation, University of Trento, (2019).

[12] Zur, L., Tran, L. T. N., Massella, D., Vaccari, A., Chiappini, A., Chiasera, A., Varas, S., Armellini, C., Carpentiero, A., Boulard, B., Dorosz, D., Pelli, S., Trono, C., Berneschi, S., Conti, G. N., Gates, J., Sazio, P. J., Rossi, B., Iacob, E., Micheli, V., Speranza, G., Ischia, G., Prudenzano, F., Lukowiak, A., Zonta, D., Ramponi, R., Righini, G. C., and Ferrari, M., "SiO $\mathrm{Si}_{2}-\mathrm{SnO}_{2}$ transparent glass-ceramics activated by rare earth ions", Proc. SPIE 10914 on Optical Components and Materials XVI, 1091411 (2019).

[13] Cascales, C., Balda, R., Lezama, L., Barredo-Zuriarrain, M., and Fernández, J., "Site symmetry and host sensitization-dependence of $\mathrm{Eu}^{3+}$ real time luminescence in tin dioxide nanoparticles", Optic Express, 26(13), 1615516170 (2018).

[14] Tran, L. T. N., Zur, L., Massella, D., Derkowska-Zielinska, B., Chiasera, A., Varas, S., Armellini, C., Martucci, A., Zonta, D., Tran, T. T. V., Lukowiak, A., Taccheo, S., Dorosz, D., Righini, G. C., Boucher, Y. G., and Ferrari, M., "SiO $2_{2}$ $\mathrm{SnO}_{2}: \mathrm{Er}^{3+}$ transparent glass-ceramics: fabrication and photonic assessment", Proc. SPIE 10683, Fiber Lasers and Glass Photonics: Materials through Applications, 106832C (2018).

[15] Tran, L. T. N., Massella, D., Zur, L., Chiasera, A., Varas, S., Armellini, C., Righini, G. C., Lukowiak, A., Zonta, D. and Ferrari, M., "SiO $2-\mathrm{SnO}_{2}: \mathrm{Er}^{3+}$ Glass-Ceramic Monoliths", Applied Sciences 8, 1335-1/8 (2018).

[16] Brinker, C. J., "Hydrolysis and condensation of silicates: effects on structure", Journal of Non-Crystalline Solids, 100(1-3), 31-50 (1988).

[17] Mounkachi, O., Salmani, E., Lakhal, M., Ez-zahraouy, H., and Hamedoun, M., "Band-gap engineering of $\mathrm{SnO}_{2}$ ”, Solar Energy Materials \& Solar Cells, 148, 34-38 (2016).

[18] Trani, F., Causà, M., Ninno, D., Cantele, G., and Barone, V., "Density functional study of oxygen vacancies at the $\mathrm{SnO}_{2}$ surface and subsurface sites", Physical Review B, 77(245410), 1-8 (2008).

[19] J. Fernández, R. Balda, C. Cascales, S. García-Revilla, "Site symmetry and host sensitization-dependence of Eu ${ }^{3+}$ real-time luminescence in tin dioxide nanoparticles", Proc. SPIE 10914, 109140T-1/12 (2019).

[20] Dong, L., Cruz, J. L., Reekie, L., Xu, M. G., Payne, D. N., "Enhanced photosensitivity in tin-codoped germanosilicate optical fibres", IEEE Photonics Technology Letters, 7(9), 1048-1050 (1995).

[21] Dong, L., Cruz, J. L., Tucknott, J. A., Reekie, L., Payne, D. N., "Strong photosensitive gratings in tin-doped phosphosilicate optical fibres", Optics Letters, 20(19), 1982-1984 (1995).

[22] Reekie, L., Dong, L., "Material considerations for Bragg fibre gratings", Proc. SPIE 2998 on Photosensitive Optical Materials and Devices, 2998:2-10 (1997).

[23] Chiodini, N,, Paleari, A., Brambilla, G., Taylor, E. R., "Erbium doped nanostructured tin-silicate glass-ceramic composites", Applied Physics Letters, 80(23), 4449-4451 (2002). 
[24] Brambilla, G., Pruneri, V., and Reekie, L., "Photorefractive index gratings in $\mathrm{SnO}_{2}: \mathrm{SiO}_{2}$ optical fibers", Applied Physics Letters 76(7), 807-809 (2000).

[25] Montero, C., Gomez-Reino, C., and Brebner, J. L., "Planar Bragg gratings made by excimer-laser modification of ion-exchanged waveguides", Optics Letters 24(21), 1487-1489 (1999).

[26] Chiodini, N., Paleari, A., Spinolo, G., Chiasera, A., Ferrari, M., Brambilla, G., and Taylor, E. R., "Photosensitive erbium doped tin-silicate glass", Journal of Non-crystalline Solids, 311(3), 217-222 (2002).

[27] Chiodini, N., Paleari, A., and Spinolo, G., "Photorefractivity in nanostructured tin-silicate glass ceramics: a radiation-induced nanocluster size effect", Physical Review Letters, 90(5):055507 (2003).

[28] Chiodini, N., Paleari, A., Spinolo, G., and Crespi P., "Photorefractivity in $\mathrm{SiO}_{2}$ : $\mathrm{SnO}_{2}$ glass-ceramics by visible light", Journal of Non-crystalline Solids, 322(1-3), 266-271 (2003). 